\title{
Higher spins tunneling from a time dependent and spherically symmetric black hole
}

\author{
Haryanto M. Siahaan ${ }^{\mathrm{a}}(\mathbb{C}$ \\ Physics Department, Parahyangan Catholic University, Jalan Ciumbuleuit 94, Bandung 40141, Indonesia
}

Received: 7 June 2015 / Accepted: 26 February 2016 / Published online: 12 March 2016

(C) The Author(s) 2016. This article is published with open access at Springerlink.com

\begin{abstract}
The discussions of Hawking radiation via tunneling method have been performed extensively in the case of scalar particles. Moreover, there are also several works in discussing the tunneling method for Hawking radiation by using higher spins, e.g. neutrino, photon, and gravitino, in the background of static black holes. Interestingly, it is found that the Hawking temperature for static black holes using the higher spins particles has no difference compared to the one computed using scalars. In this paper, we study the Hawking radiation for a spherically symmetric and time dependent black holes using the tunneling of Dirac particles, photon, and gravitino. We find that the obtained Hawking temperature is similar to the one derived in the tunneling method by using scalars.
\end{abstract}

\section{Introduction}

General relativity predicts the existence of the black holes, i.e. objects from which nothing can escape. Hawking found that incorporating quantum mechanics changes this understanding as regards black holes [1,2]. He showed that black holes are not completely black. They radiate a thermal spectrum due to the quantum effects. Furthermore, Hawking found a connection between the surface gravity of a black hole and its corresponding temperature.

There are several ways in understanding the mechanism of the black holes to radiate. One of the latest approaches was given by Parikh and Wilczek [3,4], where the radiation of the black holes is described as a quantum tunneling effect of particles through the event horizon. This method is found to be simpler and more intuitive. In the Parikh-Wilczek (PW) method, which sometimes is called the radial null geodesic method, one first computes the amplitude of tunneling across the horizon as an exponentiation of the imaginary part of the corresponding particle's action in the outgoing mode.

\footnotetext{
a e-mail: haryantoshn@gmail.com
}

Then the principle of detailed balance is used to connect the tunneling amplitude with the Boltzmann factor, hence the temperature can be obtained.

In fact, the radial null geodesic method is not the only way to describe the Hawking radiation by using the tunneling mechanism. There is an alternative, known as the Hamilton-Jacobi method [5-8], where one solves the semiclassical equation of motion for the tunneled particles by using the Hamilton-Jacobi ansatz for the corresponding particle's wave function. The treatments of the Hamilton-Jacobi method in discussing the Hawking temperature and black hole thermodynamics for various cases and black holes have been performed in [9-43]. However, most of them are confined to the tunneling of scalar fields where one starts with a Klein-Gordon equation in curved spacetime and solves it by using the Hamilton-Jacobi ansatz.

For static spacetimes, the tunneling mechanism for Hawking radiation has been extended to the case of spin $\frac{1}{2}$ fermion $[44,45]$, as well as photon and gravitino [46]. These works motivated us to study the Hawking mechanism for time dependent black hole's radiation as the tunneling processes of massless higher spins particles. The work presented in this paper can also be considered as an extension of our previous work [47] where we studied the scalar tunneling which gives rise to the Hawking temperature of Vaidya black holes. Since we keep the spherically symmetric and time dependent spacetime metric in this paper quite general, i.e. we stick to a spacetime whose line element can be written as Eq. (5), the results presented in this paper should be relevant to this class of spacetime. ${ }^{1}$

The organization of this paper is as follows. In the next section, we review the null geodesic method for a general time dependent metric background. In Sect. 3, we discuss the tunneling of massless Dirac particles across the time dependent black hole's horizon. By using the solutions of massless Dirac fields and then the detailed balance principle, we can

\footnotetext{
${ }^{1}$ For example to those discussed in [48-52].
} 
get the Hawking temperature of the black holes under consideration. Interestingly, the Hawking temperature derived in this section is invariant compared to the one obtained in the case of tunneled scalar fields [47]. The same prescription is repeated in Sect. 4, where the starting point is the source free Maxwell equation in curved space. Again, the Hamilton-Jacobi ansatz is used to solve the corresponding equation. After using the Lorenz gauge condition, we can get the solution for the vector fields. The same finding appears in Sect. 4, where the obtained Hawking temperature shows no difference from that derived in the scalar tunneling case [47].

In Sect. 5, the tunneling object is a massless gravitino, i.e. a particle with spin $\frac{3}{2}$. We start from the Rarita-Schwinger equation in curved space, and the Hamilton-Jacobi ansatz helps us to get the solutions. As we would expect after performing the analysis in Sects. 3 and 4, the Hawking radiation due to the massless gravitino tunneling yields the same temperature as the one in the scalar case [47]. The conclusions are given in the last section.

\section{Radial null geodesic method}

In [3], Parikh and Wilczek presented a direct and short derivation of Hawking radiation as a tunneling process. To dispel the coordinate singularity that the Schwarzschild coordinate has, they employ the Painlevé transformation, which yields the Schwarzschild spacetime transformation:

$\mathrm{d} s^{2}=-\left(1-\frac{2 M}{r}\right) \mathrm{d} t^{2}+2 \sqrt{\frac{2 M}{r}} \mathrm{~d} t \mathrm{~d} r+\mathrm{d} r^{2}+r^{2} \mathrm{~d} \Omega_{2}^{2}$,

where $\mathrm{d} \Omega_{2}^{2}=\mathrm{d} \theta^{2}+\sin ^{2} \theta \mathrm{d} \phi^{2}$ is the metric of a 2 -sphere with unit radius. In getting the last line element from the Schwarzschild metric, the Schwarzschild time $t_{s}$ is transformed as [3]

$t_{s}=t-2 \sqrt{2 M r}-2 M \ln \left(\frac{\sqrt{r}-\sqrt{2 M}}{\sqrt{r}+\sqrt{2 M}}\right)$.

For a general spherically symmetric and static spacetime

$\mathrm{d} s^{2}=-X(r) \mathrm{d} t^{2}+Y(r)^{-1} \mathrm{~d} r^{2}+r^{2} \mathrm{~d} \Omega_{2}^{2}$,

the Painlevé transformation (2) can be written as

$\mathrm{d} t \rightarrow \mathrm{d} t-\sqrt{\frac{1-Y}{X Y}} \mathrm{~d} r$.

In this paper we work out the tunneling prescription to explain the Hawking radiation for a time dependent and spherically symmetric black holes. In general, such black hole solutions can be found to read [53] $\mathrm{d} s^{2}=-X(t, r) \mathrm{d} t^{2}+Y(t, r)^{-1} \mathrm{~d} r^{2}+Z(t, r) \mathrm{d} \Omega_{2}^{2}$.

In this paper we study a class of a time dependent spacetime which has coordinate singularity at $r$ which yields $Y(t, r)=0$ and $X(t, r)=0$. In order to get rid of this coordinate singularity, we employ the "generalized" Painlevé transformation (4), which in differential form can be found to read

$\mathrm{d} t_{P}=\frac{\partial t_{P}}{\partial t} \mathrm{~d} t+\frac{\partial t_{P}}{\partial r} \mathrm{~d} r$.

For a general spacetime metric (5), it is clear that both $\partial t_{P} / \partial t$ and $\partial t_{P} / \partial r$ would be some functions of $t$ and $r$. However, by employing the relation which are applied in the static case, i.e. $\mathrm{d} t_{P}=\mathrm{d} t-\sqrt{\frac{1-Y}{X Y}} \mathrm{~d} r$, we find that the mappings

$\frac{\partial t_{P}}{\partial t}=1$

and

$\frac{\partial t_{P}}{\partial r}=-\sqrt{\frac{1-Y(t, r)}{X(t, r) Y(t, r)}}$

remove the coordinate singularity in the general metric (5). As in the static case, the left hand side of Eq. (8) is singular for the vanishing $X(t, r)$ or $Y(t, r)$. This is a normal consequence in such transformation to remove the coordinate singularity.

Furthermore, to get an integrable $t_{P}$, from the fact that $\frac{\partial^{2} t_{P}}{\partial r \partial t}=0$ in Eq. (7) one realizes that the right hand side of Eq. (8) must be independent of time $t$, say ${ }^{2}$

$1-Y(t, r)=X(t, r) Y(t, r) C(r)$,

where $C(r)$ is an arbitrary function of $r$. Hence, the set of the Painlevé transformation (7) and (8) works only for a class of time dependent spherically symmetric spacetimes whose metric functions satisfy the condition ${ }^{3}(9)$.

Consequently, after employing the transformation (7) and (8), the metric (5) now can be written as

$$
\begin{aligned}
\mathrm{d} s^{2}=- & X(t, r) \mathrm{d} t^{2}+2 X(t, r) \sqrt{\frac{1-Y(t, r)}{X(t, r) Y(t, r)}} \mathrm{d} t \mathrm{~d} r \\
& +\mathrm{d} r^{2}+Z(t, r) \mathrm{d} \Omega_{2}^{2} .
\end{aligned}
$$

It is understood that the coordinate singularity at $X(t, r)=$ $Y(t, r)=0$ has already been removed in (10), where instead

\footnotetext{
${ }^{2}$ In the case of static spacetime, Eq. (9) is automatically satisfied.

${ }^{3}$ In Appendix A, we show a constraint for the Vaidya black hole mass function $m(t, r)$ which comes from this condition.
} 
of being singular, now the $g_{t r}$ component of the line element above is indeterminate. ${ }^{4}$ Accordingly, the null radial geodesics from the "Painlevé transformed" metric (10) can be written as

$\frac{\mathrm{d} r}{\mathrm{~d} t}=\sqrt{\frac{X(t, r)}{Y(t, r)}}( \pm 1-\sqrt{1-Y(t, r)})$,

and the $+(-)$ signs denote the outgoing (ingoing) geodesics. Moreover, for practical benefits one can Taylor expand the coefficients $X$ and $Y$ near horizon, i.e.

$$
\left.\left.X(t, r)\right|_{t} \simeq X^{\prime}\left(t, r_{h}\right)\right|_{t}\left(r-r_{h}\right)+\left.O\left(\left(r-r_{h}\right)^{2}\right)\right|_{t}
$$

and

$$
\left.\left.Y(t, r)\right|_{t} \simeq Y^{\prime}\left(t, r_{h}\right)\right|_{t}\left(r-r_{h}\right)+\left.O\left(\left(r-r_{h}\right)^{2}\right)\right|_{t},
$$

where $r_{h}$ is the radius of the event horizon. By using the Taylor expansions (12) and (13), the outgoing null radial geodesic (11) can be approached as

$\frac{\mathrm{d} r}{\mathrm{~d} t} \simeq \frac{1}{2} \sqrt{X^{\prime}\left(r_{h}, t\right) Y^{\prime}\left(r_{h}, t\right)}\left(r-r_{h}\right)$.

Now we use the prescription by Parikh and Wilczek in getting the Hawking temperature using the picture of a particle tunneling through the event horizon, which is sometime called the radial null geodesic or the Parikh-Wilczek (PW) method. However, it was found by Chowdhury in [54] that the expression $\operatorname{Im} S=\int p_{r} \mathrm{~d} r$, which appears in the original PW method, is not canonically invariant. Therefore, we use the prescription by Akhmedov et al. [55] in computing the tunneling rate, which reads

$\Gamma \sim \exp \left[-\frac{\operatorname{Im} \oint p_{r} \mathrm{~d} r}{\hbar}\right]$

The term inside the square brackets above then can be computed as

$\operatorname{Im} \oint p_{r} \mathrm{~d} r=\operatorname{Im} \oint \int_{0}^{p_{r}} \mathrm{~d} p_{r}^{\prime} \mathrm{d} r=\operatorname{Im} \oint \int_{0}^{H} \frac{\mathrm{d} H^{\prime}}{\frac{\mathrm{d} r}{\mathrm{~d} t}} \mathrm{~d} r$,

where we have made use of the Hamilton equation $\mathrm{d} r / \mathrm{d} t=$ $\mathrm{d} H /\left.\mathrm{d} p_{r}\right|_{r}$ related to the canonical variables $r$ and $p_{r}$ (in this case, the radial component of the radius and the momentum). Different from the discussions of several authors for a static black hole mass, e.g. Refs. [3] and [56-58], the outgoing particle's energy must be time dependent for black holes with

\footnotetext{
4 We consider the case where after employing the L'Hospital's rule, one can get a non-singular form of $g_{t r}$ in the metric (5). Otherwise, the method developed in this paper might not work, since $\mathrm{d} r / \mathrm{d} t$ in (11) could be singular.
}

varying mass. So, the $\mathrm{d} H^{\prime}$ integration at (16) is for all values of the outgoing particle's energy, say from zero to $+E(t)$.

By using the approximation (14), we can perform the integration (16). For $\mathrm{d} r$ integration, we can perform a contour integration for the upper half complex plane to avoid the coordinate singularity $r_{h}$. The result is

$\operatorname{Im} \oint p_{r} \mathrm{~d} r=\frac{4 \pi E(t)}{\sqrt{X^{\prime}\left(r_{h}, t\right) Y^{\prime}\left(r_{h}, t\right)}}$.

Equalizing the tunneling rate (15) with the Boltzmann factor $\exp [-\beta E(t)]$ for a system with time dependence of energy we obtain

$T_{H}=\frac{\hbar \sqrt{X^{\prime}\left(r_{h}, t\right) Y^{\prime}\left(r_{h}, t\right)}}{4 \pi}$.

This temperature is also derived by Nielsen and Yeom in $[59,60]$ by using a slightly different approach of PW method for a general time dependent background.

At this point we understand that the temperature (18) does not care about the spin of the tunneling particles. As long as it follows the radial null geodesic, which we know is the path of massless particles, it will give the same contribution to the temperature as measured by a detector at infinity independent of the spin that it has. However, the spins that we are mentioning here are $0,1 / 2,1$ and 3/2 only, since to the best of the author's knowledge there is no work for Hawking radiation in the tunneling picture which uses spins $\geq 2$ as the tunneling particles. In the next sections, we will reproduce the temperature (18) by considering the tunneling of the Dirac fermion, photon, and gravitino from a spherically symmetric and time dependent black hole by using the Hamilton-Jacobi method.

\section{Massless Dirac particle tunneling}

In this section we will study the Hawking radiation of time dependent black holes where the tunneling particle has spin $\frac{1}{2}$. We start by writing an action describing massless Dirac fields in curved spacetime [61],

$S_{\psi}=\int d^{4} x \sqrt{-g} \bar{\Psi} i \tilde{\gamma}^{\mu}\left(\partial_{\mu}+\frac{i}{2} g^{\gamma \nu} \Gamma_{\mu \nu}^{\beta} \Sigma_{\beta \gamma}\right) \Psi$.

The corresponding equation of motion can be written as

$\tilde{\gamma}^{\mu} \nabla_{\mu} \Psi=0$

where

$\nabla_{\mu}=\partial_{\mu}+\frac{i}{2} g^{\gamma \nu} \Gamma_{\mu \nu}^{\beta} \Sigma_{\beta \gamma}$

and $\Sigma_{\alpha \beta}=\frac{i}{4}\left[\gamma_{\alpha}, \gamma_{\beta}\right]$. We use $\operatorname{diag}(-,+,+,+)$ as the Minkowski metric tensor, and the flat spacetime Dirac matri$\operatorname{ces} \gamma^{\alpha}$ are 
$\gamma^{0}=\left(\begin{array}{ll}i & 0 \\ 0 & -i\end{array}\right), \quad \gamma^{1}=\left(\begin{array}{ll}0 & \sigma^{3} \\ \sigma^{3} & 0\end{array}\right)$,

$\gamma^{2}=\left(\begin{array}{ll}0 & \sigma^{1} \\ \sigma^{1} & 0\end{array}\right), \quad \gamma^{3}=\left(\begin{array}{ll}0 & \sigma^{2} \\ \sigma^{2} & 0\end{array}\right)$.

The flat Dirac matrices $\gamma^{\alpha}$ and the "curved" ones $\tilde{\gamma}^{\mu}$ are related by $\tilde{\gamma}^{\mu}=e_{\alpha}^{\mu} \gamma^{\alpha}$.

For the general time dependent metric (5), the tetrads $e_{\mu}^{a}$ can be expressed as

$e_{\mu}^{a}=\left(\begin{array}{llll}\sqrt{X} & 0 & 0 & 0 \\ 0 & 1 / \sqrt{Y} & 0 & 0 \\ 0 & 0 & r & 0 \\ 0 & 0 & 0 & r \sin \theta\end{array}\right)$

where $g_{\mu \nu}=e_{\mu}^{a} e_{v}^{b} \eta_{a b}$. Clearly $e_{a}^{\mu}$ is just the inverse of (23). Therefore, the Dirac matrices $\tilde{\gamma}^{\mu}$ using the tetrads (23) can be written as

$\tilde{\gamma}^{t}=\frac{i}{\sqrt{X}}\left(\begin{array}{llll}1 & 0 & 0 & 0 \\ 0 & 1 & 0 & 0 \\ 0 & 0 & -1 & 0 \\ 0 & 0 & 0 & -1\end{array}\right)$,

$\tilde{\gamma}^{r}=\sqrt{Y}\left(\begin{array}{llll}0 & 0 & 1 & 0 \\ 0 & 0 & 0 & -1 \\ 1 & 0 & 0 & 0 \\ 0 & -1 & 0 & 0\end{array}\right)$,

$\tilde{\gamma}^{\theta}=\frac{1}{r}\left(\begin{array}{cccc}0 & 0 & 0 & 1 \\ 0 & 0 & 1 & 0 \\ 0 & 1 & 0 & 0 \\ 1 & 0 & 0 & 0\end{array}\right)$

$\tilde{\gamma}^{\phi}=\frac{i}{r \sin \theta}\left(\begin{array}{llll}0 & 0 & 0 & -1 \\ 0 & 0 & 1 & 0 \\ 0 & -1 & 0 & 0 \\ 1 & 0 & 0 & 0\end{array}\right)$

Now we employ the Hamilton-Jacobi ansatz for the spinor wave function describing the massless Dirac particles. To simplify the computation, we perform the tunneling process analysis for spin up particle $\Psi_{u}$ and spin down one $\Psi_{d}$ separately. ${ }^{5}$ Explicitly, the Hamilton-Jacobi ansatz for our spinors can be found to read

$\Psi_{u}=\left(\begin{array}{l}\mathcal{A} \\ 0 \\ \mathcal{B} \\ 0\end{array}\right) \exp \left(\frac{i}{\hbar} S_{u}\right)$

5 The reader is assumed to be familiar with the Dirac spinor as a direct sum, $\Psi=\Psi_{u} \oplus \Psi_{d}$.
$\Psi_{d}=\left(\begin{array}{l}0 \\ \mathcal{C} \\ 0 \\ \mathcal{D}\end{array}\right) \exp \left(\frac{i}{\hbar} S_{d}\right)$

where the function $S_{u}$ and $S_{d}$ are expanded in terms of $\hbar$ as

$S_{k}=S_{0 k}+\hbar S_{1 k}+\hbar^{2} S_{2 k}+\hbar^{3} S_{3 k}+\cdots$

and $k$ is understood as the spin of the Dirac particles under consideration, i.e. $k=u$ or $k=d$. The coefficients $\mathcal{A}, \mathcal{B}$, $\mathcal{C}$, and $\mathcal{D}$ in general are $t, r, \theta$, and $\phi$ dependent, as well as the corresponding actions for spins up and down $S_{u}$ and $S_{d}$, respectively. As in the Dirac formalism, (28) is the wave function for spin up fermion, and (29) is for the spin down. In the next step, we work out the analysis for spin up only, since an analogous method can also be performed for spin down which produces the same Hawking temperature. The case of a radial null geodesic yields the corresponding particle action not varying with respect to the $\theta$ and $\phi$ coordinates. Therefore, by inserting the spin up wave function (28) into the Eq. (20), we have a set of equations

$\frac{i \mathcal{A}}{\sqrt{X}} \partial_{t} S_{0 u}+\sqrt{Y} \mathcal{B} \partial_{r} S_{0 u}+\mathcal{O}(\hbar)=0$

$\sqrt{Y} \mathcal{A} \partial_{r} S_{0 u}-\frac{i \mathcal{B}}{\sqrt{X}} \partial_{t} S_{0 u}+\mathcal{O}(\hbar)=0$.

We focus only on the leading order terms in the equations; hence $\mathcal{O}(\hbar)$ can be neglected.

Moreover, Eqs. (31) and (32) can be shown in matrix operation terms as follows:

$$
\left(\begin{array}{ll}
i X^{-1 / 2} \partial_{t} S_{0 u} & Y^{-1 / 2} \partial_{r} S_{0 u} \\
Y^{-1 / 2} \partial_{r} S_{0 u} & -i X^{-1 / 2} \partial_{t} S_{0 u}
\end{array}\right)\left(\begin{array}{c}
\mathcal{A} \\
\mathcal{B}
\end{array}\right) \equiv \tilde{D}\left(\begin{array}{c}
\mathcal{A} \\
\mathcal{B}
\end{array}\right)=0
$$

The vanishing of the last equation is guaranteed if the determinant of $\tilde{D}$ is zero, which leads to

$\left(\partial_{t} S_{0 u}\right)^{2}=X Y\left(\partial_{r} S_{0 u}\right)^{2}$

We notice that Eq. (34) is just the equation for the scalar particle's action in curved space after we employ the HamiltonJacobi ansatz and consider only the leading terms in the equation $[5-8,47]$. Moreover, the last equation can be rewritten as

$\partial_{r} S_{0 u}= \pm \frac{1}{\sqrt{X Y}} \partial_{t} S_{0 u}$

where the $(-)+$ signs correspond to the (outgoing) ingoing modes. The discussions of these modes can be found in Appendix 2.A of [62]. 
In [47], the authors have derived the solution for an equation like (35) where they consider the tunneling of scalar particles from a time dependent black hole. Therefore, the techniques presented in [47] can be adopted to get an expression for $S_{u}$. We look for the general form of a solution for the action $^{6}$

$S_{0 u}(t, r)=-\int_{0}^{t} E\left(t^{\prime}\right) \mathrm{d} t^{\prime}+\tilde{S}_{0 u}(t, r)$,

where $E\left(t^{\prime}\right)$ stands for the time dependent energy of the Dirac particle which tunnels across the event horizon. The time dependence of the energy is understood since the mass of the black hole decreases as time passes. Taking the derivative with respect to time in both sides of the last equation provides us with

$\partial_{t} S_{0 u}(t, r)=-E(t)+\partial_{t} \tilde{S}_{0 u}(t, r)$,

and from the differentiation with respect to the radius $r$ we have

$\partial_{r} S_{0 u}(t, r)=\partial_{r} \tilde{S}_{0 u}(t, r)$

The chain rule allows us to write

$\frac{\mathrm{d} \tilde{S}_{0 u}(t, r)}{\mathrm{d} r}=\frac{\partial \tilde{S}_{0 u}(t, r)}{\partial r}+\frac{\partial \tilde{S}_{0 u}(t, r)}{\partial t} \frac{\mathrm{d} t}{\mathrm{~d} r}$.

In this section we do not use the Painlevé transformation as we have in the previous section. Therefore, the corresponding radial null geodesic in the background (5) is

$\frac{\mathrm{d} r}{\mathrm{~d} t}= \pm \sqrt{X Y}$

The $+(-)$ signs in the left hand side of (40) refers to the geodesic of outgoing (ingoing) null particles, respectively. Combining Eqs. (39) and (40) gives us

$\frac{\partial \tilde{S}_{0 u}(t, r)}{\partial r}=\frac{\mathrm{d} \tilde{S}_{0 u}(t, r)}{\mathrm{d} r} \mp \frac{1}{\sqrt{X Y}} \frac{\partial \tilde{S}_{0 u}(t, r)}{\partial t}$

with $-(+)$ signs referring to the outgoing (ingoing) spin up Dirac from the black hole.

Recall that for the dynamics of a particle with Hamiltonian $H$ and action $S$, one can show the relation [63]

$\frac{\partial S}{\partial t}+H=0$

6 A discussion of the Schrödinger equation with time dependent Hamiltonian which supports this general form of action is given in Appendix B.
The last equation also emerges in the semiclassical discussion, for example, in WKB approximation in solving the one dimensional Schrödinger equation,

$i \hbar \frac{\partial \Psi}{\partial t}=-\frac{\hbar^{2}}{2 m} \frac{\partial^{2} \Psi}{\partial x^{2}}+V(x) \Psi$

where we use the ansatz $\Psi=e^{i S / \hbar}$ and $S$ is the classical action of particle associated to the wave function $\Psi$. From Eq. (43), we may observe that the partial derivative of the action with respect to time would be a negative quantity for a particle with positive energy, since the eigenvalue of $H$ must be positive. Therefore, the (-) sign in (35) belongs to the outgoing particle,

$\partial_{t} S_{0 u}=-\sqrt{X Y} \partial_{r} S_{0 u}$,

since the momentum $p_{r}=\partial_{r} S_{0}$ is positive. Correspondingly, the one with the $(+)$ sign refers to the ingoing particle,

$\partial_{t} S_{0 u}=\sqrt{X Y} \partial_{r} S_{0 u}$

Then we use Eqs. (38), (39), (44), and (45) to get

$\frac{\mathrm{d} \tilde{S}_{0 u}(t, r)}{\mathrm{d} r}= \pm \frac{E(t)}{\sqrt{X Y}}$

whose solution can be found to read

$\tilde{S}_{0 u}(t, r)= \pm E(t) \int \frac{\mathrm{d} r}{\sqrt{X Y}}$.

The + and - signs in the last equation belong to the outgoing and ingoing particle, respectively. Accordingly, a solution for the action (36) can be found to read

$S_{0 u}(t, r)=-\int_{0}^{t} E\left(t^{\prime}\right) \mathrm{d} t^{\prime} \pm \frac{i \pi E(t)}{\sqrt{X^{\prime} Y^{\prime}}}$.

Plugging the solution (48) into (28) gives us

$\Psi_{u, i n}=\left(\begin{array}{c}\mathcal{A} \\ 0 \\ \mathcal{B} \\ 0\end{array}\right) \exp \left(\frac{i}{\hbar}\left(-\int_{0}^{t} E\left(t^{\prime}\right) \mathrm{d} t^{\prime}-\frac{i \pi E(t)}{\sqrt{X^{\prime} Y^{\prime}}}\right)\right)$

and

$\Psi_{u, \text { out }}=\left(\begin{array}{c}\mathcal{A} \\ 0 \\ \mathcal{B} \\ 0\end{array}\right) \exp \left(\frac{i}{\hbar}\left(-\int_{0}^{t} E\left(t^{\prime}\right) \mathrm{d} t^{\prime}+\frac{i \pi E(t)}{\sqrt{X^{\prime} Y^{\prime}}}\right)\right)$. 
Making the ingoing probability $P_{i n}=\left|\Psi_{u, i n}\right|^{2}$ unity, i.e. all fields that come close to a black hole will be absorbed, yields

$$
\int_{0}^{t} E\left(t^{\prime}\right) \mathrm{d} t^{\prime}=-\frac{i \pi E(t)}{\sqrt{X^{\prime} Y^{\prime}}}
$$

Therefore the outgoing probability can be written as

$$
P_{\text {out }}=\left|\Psi_{u, \text { out }}\right|^{2}=\exp \left(-\frac{4 \pi E(t)}{\hbar \sqrt{X^{\prime} Y^{\prime}}}\right) .
$$

The "detailed balance" principle tells us that

$$
P_{\text {out }}=e^{-\beta E} P_{\text {in }},
$$

which then allows us to write the Hawking temperature for a general time dependent black hole (5) as

$T_{H}=\frac{\hbar \sqrt{X^{\prime}\left(t, r_{h}\right) Y^{\prime}\left(t, r_{h}\right)}}{4 \pi}$.

The Hawking temperature (53) is interpreted as the measured temperature by a detector at infinity where the radiation consists of massless quantum particles with spin $\frac{1}{2}$ moving outward radially from the black holes.

\section{Photon tunneling}

In [46], Majhi and Samanta discuss the tunneling of a photon and gravitino, yielding the Hawking radiation from a static black hole. One of the conclusions in their work is that the Hawking radiation in the form of the tunneling of a photon and a gravitino yields the Hawking temperature which is invariant compared to the one computed in the case of scalar tunneling. In this section and the next one, we show that the same conclusion is obtained for a time dependent black hole. We start from an action for Maxwell fields in curved spacetime,

$S=-\frac{1}{4} \int \sqrt{-g} F_{\mu \nu} F^{\mu \nu} d^{4} x$

Taking the variation of $A_{\mu}$ in the action above, we obtain

$\nabla_{\mu} F^{\mu \nu}=0$

which is known as the Maxwell equation in the absence of the source $J^{v}$. By following Majhi et al. [46], we use the Hamilton-Jacobi ansatz for the vector field,

$A^{\mu} \sim k^{\mu} e^{\frac{i}{\hbar} S(t, r, \theta, \phi)}$ where $k^{\mu}$ is the polarization vector. This polarization vector is independent of the spacetime coordinates. As usual, the action is expanded as

$S(t, r, \theta, \phi)=\sum_{i=0}^{\infty} \hbar^{i} S_{i}(t, r, \theta, \phi)$

just like what we did in the spinor case (30). In [46], the polarization vector $k^{\mu}$ is also expanded in $\hbar$ since the authors discuss the quantum correction which comes from the higher order terms in $\hbar$ of $S(t, r, \theta, \phi)$ and $k^{\mu}$. However, since we are not interested in pursuing such a quantum correction, the polarization vector $k^{\mu}$ in (56) can be kept up to its semiclassical value only.

Plugging the ansatz (56) for the gauge fields into the Eq. (55), which alternatively can be expressed as

$\partial_{\mu} F^{\mu v}+\Gamma_{\tau \mu}^{\mu} F^{\tau v}+\Gamma_{\tau \mu}^{v} F^{\mu \tau}=0$

one can get

$\left(k^{v} \partial^{\mu} S_{0}-k^{\mu} \partial^{v} S_{0}\right) \partial_{\mu} S_{0}=0$.

In getting the last equation, we have taken the limit $\hbar \rightarrow 0$ in Eq. (58). We choose to work in the Lorenz gauge,

$\nabla_{\mu} A^{\mu}=0$

which, after plugging in the gauge fields (56), gives

$k^{\mu} \partial_{\mu} S_{0}=0$.

Again we have employed the limit $\hbar \rightarrow 0$ in obtaining Eq. (61). In this Lorenz gauge condition, Eq. (59) reduces to

$k^{\nu}\left(\partial^{\mu} S_{0}\right)\left(\partial_{\mu} S_{0}\right)=0$.

Working on the $t$ and $r$ sectors only in the spacetime under consideration allows us to write (62) as

$g^{t t}\left(\partial_{t} S_{0}\right)^{2}+g^{r r}\left(\partial_{r} S_{0}\right)^{2}=0$.

We find that the last equation is similar to (35) if we replace $S_{0}$ with $S_{0 u}$. It is clear since the background of spacetime where the vector probes come and fall into a black hole is also the same, i.e. the metric (5). Therefore, the solution for $S(t, r, \theta, \phi)$ for the vector fields $A_{\mu}$ can be found to read

$S_{0}(t, r)=-\int_{0}^{t} E\left(t^{\prime}\right) \mathrm{d} t^{\prime} \pm \frac{i \pi E(t)}{\sqrt{X^{\prime} Y^{\prime}}}$ 
Accordingly, the ingoing and outgoing solutions for the vector fields can be found to read

$A_{\text {in }}^{\mu} \sim k^{\mu} \exp \left(\frac{i}{\hbar}\left(-\int_{0}^{t} E\left(t^{\prime}\right) \mathrm{d} t^{\prime}-i \pi \frac{E(t)}{\sqrt{X^{\prime} Y^{\prime}}}\right)\right)$

and

$A_{\text {out }}^{\mu} \sim k^{\mu} \exp \left(\frac{i}{\hbar}\left(-\int_{0}^{t} E\left(t^{\prime}\right) \mathrm{d} t^{\prime}+i \pi \frac{E(t)}{\sqrt{X^{\prime} Y^{\prime}}}\right)\right)$,

respectively. The unit incoming probability $P_{\text {in }}=\left|A_{\text {in }}^{\mu}\right|^{2}$, the relation between incoming and outgoing probabilities,

$P_{\text {out }}=P_{\text {in }} \exp \left(-\frac{4 \pi E(t)}{\hbar \sqrt{X^{\prime} Y^{\prime}}}\right)$,

and the "detailed balance" principle $P_{\text {out }}=P_{\text {in }} \exp (-\beta E(t))$ yield the Hawking temperature,

$T_{H}=\frac{\hbar \sqrt{X^{\prime}\left(t, r_{h}\right) Y^{\prime}\left(t, r_{h}\right)}}{4 \pi}$.

One observes that the temperature in the last equation is equal to the one computed in the Dirac particle case (53) and the scalar case [47].

\section{Gravitino tunneling}

We start with the action of massless Rarita-Schwinger $\Psi_{\alpha}$ fields in curved spacetime,

$S_{\psi}=\int d^{4} x \sqrt{-g} \bar{\Psi}^{\alpha} i \tilde{\gamma}^{\mu}\left(\partial_{\mu}+\frac{i}{2} g^{\gamma \nu} \Gamma_{\mu \nu}^{\beta} \Sigma_{\beta \gamma}\right) \Psi_{\alpha}$,

where $\Sigma_{\beta \gamma}$ and the Dirac matrices $\tilde{\gamma}^{\mu}$ in the action above are those used in the Dirac action (19). Accordingly, the action (69) tells us that the corresponding equation of motion for $\Psi_{\alpha}$ can be found to read

$\tilde{\gamma}^{\mu} \nabla_{\mu} \Psi_{\alpha}=0$

which is known as the massless Rarita-Schwinger equation in curved space. It looks like the Dirac equation, with the Dirac spinor $\Psi$ is replaced by the vector-spinor $\Psi_{\mu}$. The number of degree of freedom of $\Psi_{\mu}$ is 16 , of which 8 are removed by the two additional constraints: $\tilde{\gamma}^{\mu} \Psi_{\mu}=0$ and $\nabla^{\mu} \Psi_{\mu}=0$.
The Hamilton-Jacobi ansatz for vector-spinor $\Psi_{\mu}$ can be found to read

$\Psi_{(u) \mu}=\left(\begin{array}{l}\mathcal{A}_{\mu} \\ 0 \\ \mathcal{B}_{\mu} \\ 0\end{array}\right) \exp \left(\frac{i}{\hbar} S_{(u)}\right)$

and

$\Psi_{(d) \mu}=\left(\begin{array}{l}0 \\ \mathcal{C}_{\mu} \\ 0 \\ \mathcal{D}_{\mu}\end{array}\right) \exp \left(\frac{i}{\hbar} S_{(d)}\right)$,

where $\Psi_{(u) \mu}$ and $\Psi_{(d) \mu}$ are the Rarita-Schwinger fields with spins $+3 / 2$ and $-3 / 2$, respectively. In the background (5), Eq. (70) for a radial geodesic can be found to read

$\frac{i \mathcal{A}_{\mu}}{\sqrt{X}} \partial_{t} S_{0(u)}+\sqrt{Y} \mathcal{B}_{\mu} \partial_{r} S_{0(u)}+\mathcal{O}(\hbar)=0$,

$\sqrt{Y} \mathcal{A}_{\mu} \partial_{r} S_{0(u)}-\frac{i \mathcal{B}_{\mu}}{\sqrt{X}} \partial_{t} S_{0(u)}+\mathcal{O}(\hbar)=0$.

The action $S_{0(u)}$ is understood as the zeroth order term in the action expansion $S_{(u)}=\sum_{i=0}^{\infty} \hbar^{i} S_{i(u)}$. Moreover, the last two equations are very close to (31) and (32), since the operator that applies to the massless Rarita-Schwinger fields in (70) is just the same as that applying to the massless Dirac field in (20). Analogous to the technique applied to the Dirac fermion in the previous section, we rewrite Eqs. (73) and (74) in the form

$$
\begin{aligned}
& \left(\begin{array}{ll}
i X^{-1 / 2} \partial_{t} S_{0(u)} & Y^{-1 / 2} \partial_{r} S_{0(u)} \\
Y^{-1 / 2} \partial_{r} S_{0(u)} & -i X^{-1 / 2} \partial_{t} S_{0(u)}
\end{array}\right)\left(\begin{array}{l}
\mathcal{A}_{\mu} \\
\mathcal{B}_{\mu}
\end{array}\right) \equiv \tilde{D}\left(\begin{array}{c}
\mathcal{A}_{\mu} \\
\mathcal{B}_{\mu}
\end{array}\right) \\
& \quad=0
\end{aligned}
$$

whose solution for the action $S_{0(u)}$ finally can be found as

$S_{0(u)}(t, r)=-\int_{0}^{t} E\left(t^{\prime}\right) \mathrm{d} t^{\prime} \pm \frac{i \pi E(t)}{\sqrt{X^{\prime} Y^{\prime}}}$.

The procedure that is needed in obtaining the solution (75) is obvious since we deal with the same equations as in Sect. 3 . In discussing the gravitino, we replace the complex valued functions $\mathcal{A}$ and $\mathcal{B}$ with the vectors $\mathcal{A}_{\mu}$ and $\mathcal{B}_{\mu}$, which are also complex valued. Moreover, the solutions for the spin $\frac{3}{2}$ fields then can be written as

$\Psi_{\mu(u, i n)}=\left(\begin{array}{l}\mathcal{A}_{\mu} \\ 0 \\ \mathcal{B}_{\mu} \\ 0\end{array}\right) \exp \left(\frac{i}{\hbar}\left(-\int_{0}^{t} E\left(t^{\prime}\right) \mathrm{d} t^{\prime}-\frac{i \pi E(t)}{\sqrt{X^{\prime} Y^{\prime}}}\right)\right)$, 
and

$\Psi_{\mu(u, \text { out })}=\left(\begin{array}{l}\mathcal{A}_{\mu} \\ 0 \\ \mathcal{B}_{\mu} \\ 0\end{array}\right) \exp \left(\frac{i}{\hbar}\left(-\int_{0}^{t} E\left(t^{\prime}\right) \mathrm{d} t^{\prime}+\frac{i \pi E(t)}{\sqrt{X^{\prime} Y^{\prime}}}\right)\right)$.

Again, using the "detailed balance" principle for the relation of outgoing and ingoing gravitino probabilities one can get

$T_{H}=\frac{\hbar \sqrt{X^{\prime}\left(t, r_{h}\right) Y^{\prime}\left(t, r_{h}\right)}}{4 \pi}$,

as the Hawking temperature from dynamical black holes (5) in the form of massless gravitino tunneling from black holes. We observe that the Hawking temperature due to the massless gravitino tunneling is equal to the temperatures computed in the last two sections.

\section{Conclusion and discussions}

We have analyzed the Hawking radiation in the form of a Dirac fermion, photon, and gravitino tunneling across the event horizon of time dependent black holes. The resulting Hawking temperatures are invariant, to be compared to the one obtained in the scalar tunneling case. The results lead to the conclusion that the Hawking temperature obtained in the tunneling method is independent of the spins of the tunneled particles. We confirm that for a time dependent and spherically symmetric black hole whose metric has the form (5), the Hawking temperature is independent of the spins of the tunneling particle, as in the case of a static one.

It is interesting to note that the PW method presented in Sect. 2 works for a limited case only, i.e. the spacetime metric whose metric functions satisfy the condition (9). The obtained Hawking temperature in this method is confirmed by the result derived via a Hamilton-Jacobi method by using higher spins tunneling in the succeeding sections as well as scalar tunneling [47]. In the Hamilton-Jacobi method we do not make use of the Painlevé transformation, hence there is no integrability condition (9) to be fulfilled. Such a condition also does not appear in $[59,60]$, and yet the same result for the Hawking temperature (18) is achieved by the authors. Hence, presumably there are some other transformations which yield the line element (5) so as to be regular at the event horizon, and therefore the PW method can be performed. Clearly each of these transformations will have a set of integrability conditions which justify a class of spacetime which fits in the computation of Hawking temperature using the PW method. Finding an alternative to the gen- eralized Painlevé transformation (7) and (8) which yields the metric (5) transforms to be regular at the event horizon would be challenging and we address this issue in our future work.

In their seminal paper [3], Parikh and Wilczek included the back reaction effect in their analysis of Hawking radiation in the tunneling picture. In [64], Zhang et al. carried out a study of Hawking radiation for Vaidya black holes by taking the back reaction effect into account, where the corresponding spacetime is written in the Eddington-Finkelstein coordinate rather than the Schwarzschild-like one. Moreover, we note that Zhang et al. considered the particle's energy to be time independent, unlike the time dependent case presented in this paper. Therefore, a further analysis would be interesting of the Hawking radiation for Vaidya black holes in the Schwarzschild-like geometry as presented in this paper, where the back reaction effect is considered and the particle's energy is time dependent. This is addressed in our future work.

Acknowledgments I thank Profs. Paulus Tjiang and Triyanta for useful discussions.

Open Access This article is distributed under the terms of the Creative Commons Attribution 4.0 International License (http://creativecomm ons.org/licenses/by/4.0/), which permits unrestricted use, distribution, and reproduction in any medium, provided you give appropriate credit to the original author(s) and the source, provide a link to the Creative Commons license, and indicate if changes were made.

Funded by SCOAP S $^{3}$

\section{Appendix A: Example: Vaidya black holes}

The Vaidya black hole is an interesting object [65-67]. It is an example of an exact time dependent black hole solution where it solves the Einstein equations with $T_{\mu \nu}=\rho k_{\mu} k_{\nu}$. Since $k_{\mu} k^{\mu}=0$, one can consider the Vaidya solution to describe a non-empty spacetime outside of a null radiating mass. Scalar tunneling as a mechanism for Hawking radiation from a Vaidya black hole is also discussed in [68], and the authors of $[69,70]$ study the spin $\frac{1}{2}$ tunneling as a Hawking process for this black hole.

Related to the generalized Painlevé transformation (7) and (8), we can take Vaidya black holes [47,71],

$$
\begin{aligned}
\mathrm{d} s^{2}=- & \left(\frac{\dot{m}(t, r)}{f(t, r)}\right)^{2}\left(1-\frac{2 m(t, r)}{r}\right) \mathrm{d} t^{2} \\
& +\left(1-\frac{2 m(t, r)}{r}\right)^{-1} \mathrm{~d} r^{2}+r^{2} \mathrm{~d} \Omega_{2}^{2},
\end{aligned}
$$

as a concrete example. In the last equation, $f(t, r)$ is an arbitrary function that depends on the model of mass $m(t, r)$ being used and it satisfies 
$f(t, r)=\left(1-\frac{2 m(t, r)}{r}\right) \frac{\partial m(t, r)}{\partial r}$.

It follows from Eq. (9) that the mass functions of a Vaidya black hole which are compatible with the time dependent Painlevé transformation (7) and (8) should obey an equation which reads

$2 m(t, r) f(t, r)^{2}=r \dot{m}(t, r)^{2}\left(1-\frac{2 m(t, r)}{r}\right)^{2} C(r)$.

Hence, the case of Vaidya black holes whose Hawking temperature can be computed using the method proposed in this paper are those with mass functions that satisfy Eq. (81), found to be

$$
\begin{aligned}
T_{H} & =\left\{\frac { \hbar ( m - r m ^ { \prime } ) } { 2 \pi r ^ { 2 } } \left[\left(\frac{\dot{m}}{f}\right)^{2}\right.\right. \\
& \left.\left.+\left(\frac{\dot{m}}{f}\right) \frac{\partial(\dot{m} / f)}{\partial r} \frac{r(r-2 m)}{m-r m^{\prime}}\right]^{1 / 2}\right\}_{r=r_{h}(t)} .
\end{aligned}
$$

Note that at the Schwarzschild limit [71], where $f(t, r)=$ $-\dot{m}$, the Hawking temperature above reduces to the familiar $T_{H}=\frac{\hbar}{8 \pi m}$ for a Schwarzschild black hole.

\section{Appendix B: Time dependent Hamiltonian in Schrödinger equation}

In this appendix we elaborate some subtleties in the time dependent Schrödinger equation in supporting the general form of the solution for the action (36). The analysis presented here can be found in [72], chapter 31 .

Let us consider a Hamiltonian $H(r(t))$ which describes a physical system. The variables in $r(t)$ are parameters which slowly vary with respect to time $t$. Hence, we understand that the Hamiltonian under consideration here is time dependent. Accordingly, the Schrödinger equation to be solved in this case is

$i \hbar \frac{\partial}{\partial t}|\Psi(t)\rangle=H(r(t))|\Psi(t)\rangle$.

The associated Hamiltonian operator and energy eigenvalue relation can be found to read

$H(r(t))|k, r(t)\rangle=E_{k}(r(t))|k, r(t)\rangle$,

which is valid at an instantaneous time $t$ only. The state $|k, r(t)\rangle$ is normalized accordingly to $\langle k, r(t) \mid l, r(t)\rangle=\delta_{k l}$.

The solution to Eq. (83) can be expressed as

$|\Psi(t)\rangle=\sum_{k} a_{k}(t) e^{-\frac{i}{\hbar} \int_{0}^{t} \mathrm{~d} t^{\prime} E_{k}\left(r\left(t^{\prime}\right)\right)}|k, r(t)\rangle$.

Plugging (86) into the Schrödinger equation (83) gives us

$\sum_{k} e^{-\frac{i}{\hbar} \int_{0}^{t} \mathrm{~d} t^{\prime} E_{k}\left(r\left(t^{\prime}\right)\right)}\left\{\dot{a}_{k}+a_{k} \frac{\partial}{\partial t}\right\}|k, r(t)\rangle=0$.

Using the adiabatic approximation, the solution to $a_{k}(t)$ can be found to $\mathrm{read}^{7}$

$a_{k}(t)=e^{i G_{k}(t)}$,

where $G_{k}(t)$ is some purely real time dependent function, i.e.

$G_{k}(t)=i \int_{0}^{t} \mathrm{~d} t^{\prime}\left\langle k, r\left(t^{\prime}\right)\left|\frac{\partial}{\partial t^{\prime}}\right| k, r\left(t^{\prime}\right)\right\rangle$.

Finally, the solution to the Schrödinger equation (83) in the adiabatic approximation can be found to read

$|\Psi(t)\rangle=e^{\frac{i}{\hbar} \int_{0}^{t} \mathrm{~d} t^{\prime}\left\{-E_{n}\left(r\left(t^{\prime}\right)\right)+\hbar\left\langle k, r\left(t^{\prime}\right)\left|\frac{\partial}{\partial t^{\prime}}\right| k, r\left(t^{\prime}\right)\right\rangle\right\}}|k, r(t)\rangle$,

which justifies the general form of the action's solution (36).

\section{References}

1. S.W. Hawking, Nature 248, 30 (1974)

2. S.W.Hawking, Commun. Math. Phys. 43, 199 (1975) [Erratumibid. 46, 206 (1976)]

3. M.K. Parikh, F. Wilczek, Phys. Rev. Lett. 85, 5042 (2000)

4. M.K. Parikh, Int. J. Mod. Phys. D 13, 2351 (2004)

5. K. Srinivasan, T. Padmanabhan, Phys. Rev. D 60, 024007 (1999)

6. S. Shankaranarayanan, K. Srinivasan, T. Padmanabhan, Mod. Phys. Lett. A 16, 571 (2001)

7. S. Shankaranarayanan, T. Padmanabhan, K. Srinivasan, Class. Quantum Grav. 19, 2671 (2002)

8. S. Shankaranarayanan, Phys. Rev. D 67, 084026 (2003)

9. S. Hemming, E.K. Vakkuri, Phys. Rev. D 64, 044006 (2001)

10. E.C. Vagenas, Phys. Lett. B 559, 65 (2003)

11. M. Angheben, M. Nadalini, L. Vanzo, S. Zerbini, JHEP 0505, 014 (2005)

12. A.J.M. Medved, E.C. Vagenas, Mod. Phys. Lett. A 20, 2449 (2005)

13. M. Arzano, A.J.M. Medved, E.C. Vagenas, JHEP 0509, 037 (2005)

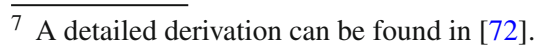


14. P. Mitra, Phys. Lett. B 648, 240 (2007)

15. E.C. Vagenas, Nuovo Cim. B 117, 899 (2002)

16. E.C. Vagenas, Mod. Phys. Lett. A 17, 609 (2002)

17. S.P. Kim, JHEP 0711, 048 (2007)

18. B.D. Chowdhury, Pramana 70, 3 (2008)

19. E.T. Akhmedov, V. Akhmedova, D. Singleton, Phys. Lett. B 642 , 124 (2006)

20. E.T. Akhmedov, V. Akhmedova, D. Singleton, T. Pilling, Int. J. Mod. Phys. A 22, 1705 (2007)

21. T. Pilling, Phys. Lett. B 660, 402 (2008)

22. Qing-Quan Jiang, Wu Shuang-Qing, Xu Cai, Phys. Rev. D 73, 064003 (2006)

23. Hu Yapeng, Jingyi Zhang, Zheng Zhao, Mod. Phys. Lett. A 21, $2143(2006)$

24. Xu Zhibo, Bin Chen, Phys. Rev. D 75, 024041 (2007)

25. Qing-Quan Jiang, Wu Shuang-Qing, Phys. Lett. B 635, 151 (2006)

26. Hu Yapeng, Jingyi Zhang, Zheng Zhao, Int. J. Mod. Phys. D 16, 847 (2007)

27. S. Stotyn, K. Schleich, B. Witt, Class. Quant. Grav. 26, 065010 (2009)

28. J. Zhang, Phys. Lett. B 668, 353 (2008)

29. K. Nozari, S.H. Mehdipour, Class. Quan. Grav. 25, 175015 (2008)

30. S. Zhou, W. Liu, Phys. Rev. D 77, 104021 (2008)

31. Z.Z. Ma, Phys. Lett. B 666, 376 (2008)

32. K. Chiang, S.M. Ke, D.T. Peng, T. Feng, Chin. Phys. Lett. 26, 070401 (2009)

33. B. Zhang, Q. Cai, M.S. Zhan, Phys. Lett. B 671, 310 (2009)

34. R.G. Cai, L.-M. Cao, Y.-P. Hu, Class. Quant. Grav. 26, 155018 (2009)

35. R. Banerjee, B.R. Majhi, Phys. Lett. B 675, 243 (2009)

36. R. Banerjee, B.R. Majhi, Phys. Lett. B 662, 62 (2008)

37. R. Banerjee, B.R. Majhi, JHEP 0806, 095 (2008)

38. R. Banerjee, B.R. Majhi, Phys. Lett. B 674, 218 (2009)

39. B.R. Majhi, Phys. Lett. B 686, 49 (2010)

40. R. Banerjee, B.R. Majhi, E.C. Vagenas, Phys. Lett. B 686, 279 (2010)

41. R. Banerjee, B.R. Majhi, E.C. Vagenas, Europhys. Lett. 92, 20001 (2010)

42. R. Banerjee, B.R. Majhi, S. Samanta, Phys. Rev. D 77, 124035 (2008)

43. R. Banerjee, B.R. Majhi, Phys. Rev. D 79, 064024 (2009)
44. R. Kerner, R.B. Mann, Class. Quant. Grav. 25, 095014 (2008)

45. R. Kerner, R.B. Mann, Phys. Lett. B 665, 277 (2008)

46. B.R. Majhi, S. Samanta, Ann. Phys. 325, 2410 (2010)

47. H.M. Siahaan, Int Triyanta, J. Mod. Phys A25, 1 (2010)

48. T. Zhu, J.R. Ren, M.F. Li, JCAP 0908, 010 (2009)

49. K.X. Jiang, T. Feng, D.T. Peng, Int. J. Theor. Phys. 48, 2112 (2009)

50. T. Zhu, J.R. Ren, Eur. Phys. J. C 62, 413 (2009)

51. S. Chakraborty, S. Saha, Adv. High Energy Phys. 2014, 168487 (2014)

52. L. Vanzo, G. Acquaviva, R. Di Criscienzo, Class. Quant. Grav. 28, $183001(2011)$

53. S. Weinberg, Gravitation and Cosmology: Principles and Applications of the General Theory of Relativity (Wiley, New York, 1972), p. 688

54. B.D. Chowdhury, Pramana 70, 593 (2008)

55. E.T. Akhmedov, T. Pilling, D. Singleton, Int. J. Mod. Phys. D 17, $2453(2008)$

56. K. Matsuno, K. Umetsu, Phys. Rev. D 83, 064016 (2011)

57. K. Lin, S. Yang, Phys. Lett. B 680, 506 (2009)

58. K. Umetsu, Int. J. Mod. Phys. A 25, 4123 (2010)

59. A.B. Nielsen, D.H. Yeom, Int. J. Mod. Phys. A 24, 5261 (2009)

60. A.B. Nielsen, J.H. Yoon, Class. Quant. Grav. 25, 085010 (2008)

61. M. Nakahara, Geometry, Topology, and Physics (Institute of Physics Publishing, Bristol, 1989)

62. B.R. Majhi, Quantum Tunneling in Black Holes. Ph.D. Thesis, University of Calcutta (2010). arXiv: 1110.6008 [gr-qc]

63. H. Goldstein, C.P. Poole, J.L. Safko, Classical Mechanics, 3rd edn. (Addison-Wesley, Boston, 2001)

64. Y. Zhang, W. Liu, Astrophys. Space Sci. 312, 315 (2007)

65. S.H. Mehdipour, Can. J. Phys. 90, 425 (2012)

66. M. Sharif, W. Javed, J. Exp. Theor. Phys. 114, 933 (2012)

67. S.H. Mehdipour, Phys. Rev. D 81, 124049 (2010)

68. J. Ren, J. Zhang, Z. Zhao, Chin. Phys. Lett. 23, 2019 (2006)

69. H.L. Li, S.Z. Yang, T.J. Zhou, R. Lin, Europhys. Lett. 84, 20003 (2008)

70. K. Lin, S. Yang, Mod. Phys. Lett. A 24, 1775 (2009)

71. P.C. Vaidya, Proc. Indian Acad Sci. A 33, 264 (1951). Reprinted in Gen. Rel. Grav. 31 (1999)

72. W. Dittrich, M. Reuter, Classical and Quantum Dynamics: From Classical Paths to Path Integrals (Springer, Berlin, 1992) 